\title{
A series involving Catalan numbers: Proofs and demonstrations
}

\author{
T. Amdeberhan, X. Guan, L. Jiu, V.H. Moll and C. Vignat
}

\begin{abstract}
Tewodros Amdeberhan obtained his Ph.D. in mathematics from Temple University under the supervision of Doron Zeiberger. He currently teaches at Tulane University and holds a permanent membership at DIMACS, Rutgers University.

Xiao Guan was born in Beijing, China. He is currently a graduate student working under the guidance of V. Moll at Tulane University. Inspired by his advisor, he is currently concentrating on problems related to the evaluation of definite integrals. He is also interested in random matrices.

Lin Jiu is a doctoral student working under the supervision of V. Moll at Tulane University. He obtained both bachelor and master degrees in Mathematics at Beijing Institute of Technology. His research interests involve Symbolic Computations, Special Functions and Number Theory.

Victor H. Moll is a professor of Mathematics at Tulane University. He was a student at the Courant Institute, working under the supervision of Henry P. McKean. His areas of interest include Special Functions, Number Theory and Probability. He enjoys collaborating with undergraduate and graduate students.

Christophe Vignat received his BS degree in Engineering at Ecole Supérieure d'Electricité and obtained his Ph.D. at Université Paris-Sud in Orsay, France. He is a professor at the Physics Department of Université Paris-Sud in Orsay, and visiting professor at Tulane University. His research interests include special functions, probability theory and number theory.
\end{abstract}

Die Catalan-Zahlen tauchen nicht nur bei zahlreichen kombinatorischen Problemen auf, sie haben auch vielfältige zahlentheoretische und analytische Eigenschaften. So wurde in einer kürzlich erschienenen Ausgabe des American Mathematical Monthly nach dem Wert bestimmter Reihen gefragt, bei denen die Catalan-Zahlen im Nenner auftreten. Über diese Reihen werden die Catalan-Zahlen mit der Zahl $\pi$ verknüpft. Die Autoren der vorliegenden Arbeit verallgemeinern das Problem und finden einen geschlossenen Ausdruck für die erzeugende Funktion der reziproken Catalan-Zahlen. Da verschiedene Beweise angeboten werden, ist für jeden Geschmack etwas dabei: Eine Differentialgleichung, das Gaußsche Fehlerintegral, die Mellin-Transformation und Bessel-Funktionen. Dabei wird auch die Rolle von Computeralgebrasystemen in derartigen Betrachtungen beleuchtet. 


\section{Introduction}

In a recent issue of The American Mathematical Monthly, the readers will find

Problem 11765. Proposed by David Beckwith, Sag Harbor, NY. Let $C_{n}$ be the $n$th Catalan number, defined by $C_{n}=\frac{1}{n+1}\left(\begin{array}{c}2 n \\ n\end{array}\right)$.

Show that

$$
\sum_{n=0}^{\infty} \frac{2^{n}}{C_{n}}=5+\frac{3 \pi}{2} \quad \text { and } \quad \sum_{n=0}^{\infty} \frac{3^{n}}{C_{n}}=22+8 \sqrt{3} \pi .
$$

The question can be made slightly more challenging by asking:

Modified problem 11765. Find the values of the series

$$
\sum_{n=0}^{\infty} \frac{2^{n}}{C_{n}} \quad \text { and } \quad \sum_{n=0}^{\infty} \frac{3^{n}}{C_{n}}
$$

It is often the case that the solution of a problem becomes easier if one becomes more ambitious and aims to answer a more general question. In this context, we ask:

Generalized question. Find a closed-form formula for

$$
f(z)=\sum_{n=0}^{\infty} \frac{z^{n}}{C_{n}}
$$

The ratio test shows that the series converges for $|z|<4$, this will be reflected in the expressions obtained for $f(z)$. The original questions correspond to the values $f(2)$ and $f(3)$. The reader will find a nice introduction to Catalan numbers in [9].

The results presented here come from our effort to produce different formulations in solving the problem at hand. The goal is to use a variety of methods that illustrate the approach that the authors use in the evaluation of series and integrals. The original question is relatively simple, so it is a perfect example to motivate these methods. The reader will travel to the world of Special Functions, Symbolic Computational Systems/Languages and Automatic Proofs. Many of the solutions will lead to the evaluation of definite integrals. Aside from classical and symbolic methods, the authors have chosen the table of integrals by I.S. Gradshteyn and I.M. Ryzhik [6] as the main source for definite integrals. The reliability of the entries in this table is of interest to the fourth author.

The word Show in Problem 11765 is interpreted here as Prove, so it is required to have a preliminary discussion on what constitutes a mathematical proof. A search in the literature reveals a large variety of documents involving a discussion on this topic. For instance, lecture notes for an Introduction to mathematical arguments by Michael Hutchings, begins with the following statement: A mathematical proof is an argument which convinces other people that something is true. A colloquial mathematical joke on this topic is: 'You only need to convince three people: one editor and two referees'. Henry McKean [11, p. 104] provides a quote attributed to Mark Kac: 'A demonstration is to 
convince a reasonable man, a proof is to convince an unreasonable man'. Under this point of view, most of the arguments presented here fall under the category of demonstrations. The reader will find in [2] and [17] some discussions on the role of computers in proofs, [7] and [8] describe the role of proof in Mathematics and Physics, [14] describes the role of proof in progress in Mathematics and [5] presents an analysis on the role of proofs in the classroom environment.

\section{The first proof: a Mathematica evaluation}

In the second decade of the 21 st century, it is natural to approach the question above as follows: What does a Computer Algebra system say about the value of this series? The authors use Mathematica and in version 9.0 simply input the line in boldface below to obtain the answer.

$$
\begin{aligned}
\operatorname{In}[1]:= & \text { Sum }\left[2^{n} / \text { CatalanNumber }[n],\{n, 0, \text { Infinity }\}\right] / / \text { Expand } \\
\text { Out }[1] & =5+3 \pi / 2
\end{aligned}
$$

The command Expand simply transforms the answer from $\frac{1}{2}(10+3 \pi)$ to the form stated above. This gives the first evaluation. A similar Mathematica calculation gives the second one, this time the command Function Expand is used in the simplification. Is this considered a mathematical proof? There is a variety of sophisticated, well-tested algorithms behind the evaluation presented above. Given enough time, one could run the algorithm by hand and verify each of the steps. Would that constitute a proof?

\section{The generalization}

In the process of solving a question, such as the one proposed here, the authors always keep in mind how to explain their approach to students. The statement of the current problem leads naturally to the following question: Is it possible to replace the values 2 and 3 by a general variable? In other words, is it possible to produce a closed form for the generating function

$$
f(z)=\sum_{n=0}^{\infty} \frac{z^{n}}{C_{n}} .
$$

This is the generalized question mentioned in Section 1.

Using Mathematica again,

$\operatorname{In}[2]:=\operatorname{Sum}\left[z^{n} /\right.$ CatalanNumber $[n],\{n, 0$, Infinity $\left.\}\right]$

Out[2]= Hypergeometric [1, 2, 1/2, $z / 4]$.

The answer now involves the hypergeometric function

$$
{ }_{2} F_{1}\left(\begin{array}{c|c}
a, b & z \\
c & \mid
\end{array}\right)=\sum_{n=0}^{\infty} \frac{(a)_{n}(b)_{n}}{(c)_{n} n !} z^{n}
$$

with

$$
(a)_{n}=a(a+1)(a+2) \cdots(a+n-1)
$$


the Pochhammer symbol. These might be expressed in terms of the gamma function

$$
\Gamma(s)=\int_{0}^{\infty} t^{s-1} e^{-t} d t, \quad \text { for } \operatorname{Re} s>0
$$

as

$$
(a)_{n}=\frac{\Gamma(a+n)}{\Gamma(n)}
$$

The reader will find in [3] extensive information about this special function.

To the non-expert, the use of a symbolic package to evaluate a series, has had a positive effect: it lead him/her to one of the most beautiful non-elementary functions. For a teacher of Mathematics, this is great. Naturally, this brings many questions, the most basic of which is whether the expression for the generating function (3.1) can be simplified.

A power series $h(z)=\sum_{n=0}^{\infty} u_{n} z^{n}$ is called hypergeometric if the ratio $\frac{u_{n+1}}{u_{n}}$ is a rational function of $n$. Most functions encountered in elementary courses are hypergeometric. The exponential $h(z)=e^{z}$ is one of them, since in this case $u_{n}=\frac{1}{n !}$ and $\frac{u_{n+1}}{u_{n}}=\frac{1}{n+1}$ is a rational function of $n$. Naturally, the geometric series, for which $u_{n}=1$ is also hypergeometric function. The canonical notation for these functions comes from the factorization

$$
\frac{u_{n+1} z^{n+1}}{u_{n} z^{n}}=\frac{\left(n+a_{1}\right)\left(n+a_{2}\right) \cdots\left(n+a_{p}\right)}{\left(n+b_{1}\right)\left(n+b_{2}\right) \cdots\left(n+b_{q}\right)} \frac{\alpha z}{n+1}
$$

where $\left\{-a_{j}: 1 \leq j \leq p\right\}$ are the zeros of the numerator and $\left\{-b_{j}: 1 \leq j \leq q\right\}$ are the zeros of the denominator. The constant $\alpha$ comes from the leading coefficients in the factorization of the rational function. The convention is to always include the factor $n$ ! in the form of the series and write it as $h(z)=\sum_{n=0}^{\infty} u_{n} \frac{z^{n}}{n !}$. This can be accomplished by adjusting the definition of $u_{n}$ and to obtain the term $n+1$ in the denominator of (3.6). The notation is

$$
h(z)={ }_{p} F_{q}\left(\begin{array}{llll}
a_{1} & a_{2} & \cdots & a_{p} \\
b_{1} & b_{2} & \cdots & b_{q}
\end{array} \mid \alpha z\right) .
$$

As a second example of the representation of an elementary function, the reader can check directly that

$$
\frac{\operatorname{ArcTan} z}{z}={ }_{2} F_{1}\left(\begin{array}{cc}
\frac{1}{2} & 1 \\
\frac{3}{2} & \mid-z^{2}
\end{array}\right) .
$$

To get the power series expansion of $\operatorname{ArcTan} z$, expand $1 /\left(1+z^{2}\right)$ as a geometric series and integrate term by term.

The hypergeometric representation for the generating function $f(z)$ in (3.1) comes from identifying

$$
u_{n}=\frac{n !}{C_{n}}
$$


and the computation

$$
\frac{u_{n+1} z^{n+1}}{u_{n} z^{n}}=\frac{(n+1)(n+2)}{n+\frac{1}{2}} \frac{z}{4}
$$

from which one can read the zeros $a_{1}=1, a_{2}=2$ and the pole $b_{1}=\frac{1}{2}$ to obtain

$$
f(z)={ }_{2} F_{1}\left(\begin{array}{c|c}
1,2 & z \\
\frac{1}{2} & \frac{z}{4}
\end{array}\right) .
$$

This confirms the evaluation given by Mathematica.

\section{A simplification}

Now that a hypergeometric expression for the generating function has been produced, it remains to explore the possibility of transforming it to simpler functions. This is, in general, a complicated process. Once more, we return to Mathematica for help.

The direct command yields the expected answer:

$$
\begin{aligned}
& \operatorname{In}[3]:=\text { Hypergeometric } 2 F 1[1,2,1 / 2, z / 4] / / \text { FunctionExpand // FullSimplify } \\
& \text { Out }[3]=\frac{2}{(z-4)^{2}}\left(z+8+\frac{12 \sqrt{z}}{\sqrt{4-z}} \operatorname{ArcCsc}\left[\frac{2}{\sqrt{z}}\right]\right) .
\end{aligned}
$$

This can be expressed as

$$
f(z)=\frac{2}{(z-4)^{4}}\left(z+8+\frac{12 \sqrt{z}}{\sqrt{4-z}} \operatorname{ArcSin}\left(\frac{\sqrt{z}}{2}\right)\right) .
$$

The singularity at $z=4$, coming from the radius of convergence of the series, can be seen in this formula.

The values stated in the problem now follow from this formula. An elementary proof of (4.1) is presented next.

The first part of the argument is to produce a recurrence for the coefficients $1 / C_{n}$. This has already appeared in the computation of the ratio (3.10):

$$
(n+2) C_{n+1}=2(2 n+1) C_{n} .
$$

Then

$$
\begin{aligned}
f(z) & =\sum_{n=0}^{\infty} \frac{z^{n}}{C_{n}}=\sum_{n=0}^{\infty} \frac{2(2 n+1)}{n+2} \frac{z^{n}}{C_{n+1}} \\
& =4 \sum_{n=0}^{\infty} \frac{z^{n}}{C_{n+1}}-6 \sum_{n=0}^{\infty} \frac{z^{n}}{(n+2) C_{n+1}} .
\end{aligned}
$$


The goal is to express the right-hand side in terms of $f(z)$. As a first step, multiply by $z$ to obtain

$$
z f(z)=4 \sum_{n=1}^{\infty} \frac{z^{n}}{C_{n}}-6 \sum_{n=1}^{\infty} \frac{z^{n}}{(n+1) C_{n}}
$$

and multiplying by $z$ one more time gives

$$
z^{2} f(z)-4 z f(z)+4 z=-6 \sum_{n=1}^{\infty} \frac{z^{n+1}}{(n+1) C_{n}}
$$

The second step is to eliminate the term $n+1$ in the denominator. This is accomplished by differentiation. It follows that

$$
z(z-4) f^{\prime}(z)+2(z+1) f(z)=2 .
$$

This is a non-homogeneous first-order differential equation. To solve it, multiply by the integrating factor $(4-z)^{5 / 2} z^{-1 / 2}$ (this is the integral of the exponential of the quotient of $2(z+1)$ by $z(z-4))$ to transform the left-hand side of (4.3) into a perfect derivative. Then integrate to obtain the result.

Theorem 4.1. The generating function of the reciprocal of Catalan numbers is given by

$$
f(z)=\frac{2(z+8)}{(4-z)^{2}}+\frac{24 \sqrt{z}}{(4-z)^{5 / 2}} \operatorname{ArcSin}\left(\frac{\sqrt{z}}{2}\right) .
$$

Naturally, the length of a solution to a mathematical question depends on what is assumed by the authors. Readers familiar with [10], will know the power series expansion

$$
\sum_{n=0}^{\infty} \frac{z^{2 n}}{\left(\begin{array}{c}
2 n \\
n
\end{array}\right)}=\frac{4}{4-z^{2}}\left[1+\frac{z \operatorname{ArcSin}(z / 2)}{\sqrt{4-z^{2}}}\right] .
$$

To obtain the generating function (4.4), replace $z^{2}$ by $z$, multiply by $z$ and differentiate.

\section{A direct evaluation of $f(z)$}

The starting point of a proof can be crucial in determining its length. This section presents an evaluation of the series (3.1) based on an integral representation for the reciprocal of the central binomial coefficients

$$
\frac{1}{\left(\begin{array}{c}
2 n \\
n
\end{array}\right)}=\frac{n ! n !}{(2 n) !}=\frac{\Gamma^{2}(n+1)}{\Gamma(2 n+1)} .
$$

This proof also appears in [13].

The basic connection

$$
B(x, y)=\frac{\Gamma(x) \Gamma(y)}{\Gamma(x+y)}
$$


with the beta function defined by

$$
B(x, y)=\int_{0}^{1} t^{x-1}(1-t)^{y-1} d t
$$

yields

$$
\frac{1}{\left(\begin{array}{c}
2 n \\
n
\end{array}\right)}=n B(n, n+1) \text {. }
$$

This identity appears in [4, Exercise 10.2.2, p. 193].

Summing (5.4) for $n=1,2, \ldots$ and interchanging the series with the integral yields

$$
\sum_{n=1}^{\infty} \frac{z^{n}}{\left(\begin{array}{c}
2 n \\
n
\end{array}\right)}=\sum_{n=1}^{\infty} n z^{n} \int_{0}^{1} t^{n}(1-t)^{n} \frac{d t}{t}=\int_{0}^{1} \frac{z(1-t) d t}{[1-z t(1-t)]^{2}}
$$

This last integral can be evaluated by partial fractions to produce (4.5). The generating function (3.1) is now obtained as before.

\section{A connection with the error function}

This section presents another evaluation of the generating function $f(z)$ in $(1.1)$ based on the error function

$$
\operatorname{erf}(z)=\frac{2}{\sqrt{\pi}} \int_{0}^{z} e^{-t^{2}} d t
$$

encountered by the reader in basic Probability courses.

The story begins with a modification of the generating function for $f(z)$ defined by

$$
h(z)=\sum_{n=0}^{\infty} \frac{1}{C_{n}} \frac{z^{n}}{n !}=\sum_{n=0}^{\infty} \frac{(n+1) !}{(2 n) !} z^{n} .
$$

The relation between $f(z)$ and $h(z)$ is part of a general result of power series described next.

The standard notation for the coefficient of $z^{n}$ in the expansion of the power series for a function $R(z)$ is denoted by $\left[z^{n}\right] R$. The next statement is of Laplace transform type and it is obtained by term by term integration of the series on the right-hand side.

Lemma 6.1. Assume $A(z)=\int_{0}^{\infty} e^{-t} B(z t) d t$. Then $\left[z^{n}\right] A=n ! \times\left[z^{n}\right] B$.

Now apply this lemma to the function $h(z)$ in (6.2) to obtain an expression for $f(z)$. The integral in Lemma 6.1 is evaluated first using a symbolic language. Indeed, Mathematica gives

$$
h(z)=1+\frac{z}{4}+\frac{1}{8} e^{z / 4} \sqrt{\pi z}(z+6) \operatorname{erf}\left(\frac{\sqrt{z}}{2}\right) .
$$


This can be verified using the expansion

$$
\operatorname{erf}(z)=\frac{2 z}{\sqrt{\pi}}{ }_{1} F_{1}\left(\begin{array}{c}
\frac{1}{2} \\
\frac{3}{2}
\end{array} \mid-z^{2}\right)
$$

Lemma 6.1 now gives

$$
f(z)=\int_{0}^{\infty} e^{-t}\left(1+\frac{z t}{4}+\frac{1}{8} e^{z t / 4} \sqrt{\pi z t}(z t+6) \operatorname{erf}\left(\frac{\sqrt{z t}}{2}\right)\right) d t .
$$

The first two terms can be integrated in elementary terms. To simplify (6.5), make the change of variables $u=\sqrt{z t} / 2$ and replace $\operatorname{erf}(u)$ by $1-\operatorname{erfc}(u)$, where erfc is the complementary error function and then introduce the notation

$$
H_{n, m}(b)=\int_{0}^{\infty} x^{n} e^{-b x^{2}}[\operatorname{erfc}(x)]^{m} d x
$$

After the computation of some elementary integrals (6.5) becomes

$$
f(z)=1+\frac{z}{4}+\frac{12 \pi \sqrt{z}}{(4-z)^{5 / 2}}-\frac{4 \sqrt{\pi}}{z}\left[2 H_{4,1}(4 / z-1)+3 H_{2,1}(4 / z-1)\right] .
$$

The integrals $H_{n, m}(b)$ have been discussed in [1]. There the reader will find the values

$$
H_{2,1}(b)=\frac{1}{2 b \sqrt{\pi}}\left(\frac{\operatorname{Arctan} \sqrt{b}}{\sqrt{b}}-\frac{1}{b+1}\right)
$$

and

$$
H_{4,1}(b)=\frac{1}{b \sqrt{\pi}}\left(\frac{3 \operatorname{Arctan} \sqrt{b}}{4 b^{3 / 2}}-\frac{3}{4 b(b+1)}-\frac{1}{2(b+1)^{2}}\right) .
$$

Replacing these evaluations in (6.7) gives (4.4).

\section{A Mellin transform approach}

Start with the expansion

$$
A(z)=\sum_{n=0}^{\infty} \frac{z^{n}}{(2 n) !}=\cosh \sqrt{z}
$$

and interpret the Gamma integral

$$
\Gamma(n)=\int_{0}^{\infty} x^{n-1} e^{-x} d x
$$

as the Mellin transform

$$
\mathcal{M}[h](s)=\int_{0}^{\infty} x^{s-1} h(x) d x
$$


of the exponential function $h(x)=e^{-x}$ on $\mathbb{R}^{+}$computed at $s=n$. Since

$$
f(z)=\sum_{n=0}^{\infty} \frac{z^{n}}{(2 n) !} n !(n+1) !,
$$

we compute

$$
n !(n+1) !=\mathcal{M}\left[e^{-x}\right] \mathcal{M}\left[x e^{-x}\right](n+1)
$$

and apply the Mellin convolution theorem

$$
\mathcal{M}\left[h_{1}(x)\right] \mathcal{M}\left[h_{2}(x)\right](s)=\mathcal{M}[h(x)](s)
$$

with

$$
h(x)=\int_{0}^{\infty} h_{1}\left(x_{1}\right) h_{2}\left(\frac{x}{x_{1}}\right) \frac{d x_{1}}{x_{1}} .
$$

In our case, this gives

$$
h(x)=\int_{0}^{\infty} e^{-x_{1}} \frac{x}{x_{1}} e^{-\frac{x}{x_{1}}} \frac{d x_{1}}{x_{1}}=2 \sqrt{x} K_{1}(2 \sqrt{x}),
$$

where $K_{v}(z)$ is the modified Bessel function of the second kind with integral representation

$$
K_{v}(z)=\frac{1}{2}\left(\frac{z}{2}\right)^{v} \int_{0}^{\infty} \frac{e^{-\left(t+\frac{z^{2}}{4 t}\right)}}{t^{v+1}} d t
$$

appearing as entry 8.432 .6 in [6]. Now

$$
\begin{aligned}
f(z) & =\sum_{n=0}^{\infty} \frac{z^{n}}{(2 n) !} \mathcal{M}[h(x)](n)=\sum_{n=0}^{\infty} \frac{z^{n}}{(2 n) !} \int_{0}^{\infty} h(t) t^{n} d t \\
& =\int_{0}^{\text {infty }} h(t) \cosh \sqrt{t z} d t
\end{aligned}
$$

gives the generating function $f(z)$ as a formidable integral

$$
f(z)=\int_{0}^{\infty} \cosh \sqrt{t z} 2 \sqrt{t} K_{1}(2 \sqrt{t}) d t .
$$

The simpler looking version

$$
f(z)=\frac{1}{2} \int_{0}^{\infty} t^{2} \cosh (\gamma t) K_{1}(t) d t, \text { with } \gamma=\frac{1}{2} \sqrt{z},
$$

is obtained by the natural change of variables $\sqrt{t} \mapsto t$.

The remainder of this section is dedicated to its evaluation. First observe that Mathematica gives

$$
\frac{1}{2} \int_{0}^{\infty} t^{2} \cosh (\gamma t) K_{1}(t) d t=\frac{2+\gamma^{2}}{2\left(1-\gamma^{2}\right)^{2}}+\frac{3 \gamma}{2\left(1-\gamma^{2}\right)^{5 / 2}} \operatorname{ArcSin} \gamma
$$

and, with $\gamma=\sqrt{z} / 2$, this produces (4.5). Symbolic languages do perform. 
The authors now propose the following challenge: produce a proof of the formula (7.1) using only the entries appearing in the table of integrals [6]. Aside from promoting this table, the restriction is meant to reflect the way the authors work: given an integral, the first step is to check if it appears in this table.

To begin with, the integral is a linear combination of entry 6.621.3:

$$
\begin{aligned}
\int_{0}^{\infty} t^{\mu-1} e^{-\alpha t} K_{v}(\beta t) d t \\
\quad=\frac{\sqrt{\pi}(2 \beta)^{v}}{(\alpha+\beta)^{\mu+1}} \frac{\Gamma(\mu+v) \Gamma(\mu-v)}{\Gamma\left(\mu+\frac{1}{2}\right)}{ }_{2} F_{1}\left(\begin{array}{c}
\mu+v, v+\frac{1}{2} \mid \frac{\alpha-\beta}{\alpha+\beta} \\
\mu+\frac{1}{2}
\end{array}\right),
\end{aligned}
$$

that in the special case $\mu=3, v=1$ and $\beta=1$ gives

$$
\int_{0}^{\infty} t^{2} e^{-\alpha t} K_{1}(t) d t=\frac{32}{5(\alpha+1)^{4}} 2 F_{1}\left(\begin{array}{c}
4, \frac{3}{2} \\
\frac{7}{2}
\end{array} \mid \frac{\alpha-1}{\alpha+1}\right) .
$$

The relation $\cosh u=\frac{1}{2}\left(e^{u}+e^{-u}\right)$ and the expression (3.11) show that the evaluation of the generating function $f(z)$ is equivalent to the hypergeometric identity

$$
\begin{aligned}
& { }_{2} F_{1}\left(\begin{array}{c|c}
1,2 & z \\
\frac{1}{2} & \frac{1}{4}
\end{array}\right)=\frac{128}{5}\left[\frac{1}{(\sqrt{z}+2)^{4}}{ }^{2} F_{1}\left(\begin{array}{c}
4, \frac{3}{2} \\
\frac{7}{2}
\end{array} \mid \frac{\sqrt{z}-2}{\sqrt{z}+2}\right)\right. \\
& \left.+\frac{1}{(\sqrt{z}-2)^{4}} F_{1}\left(\begin{array}{c}
4, \frac{3}{2} \mid \frac{\sqrt{z}+2}{2} \\
\frac{7}{2}
\end{array}\right)\right] .
\end{aligned}
$$

The proof of this identity begins with an application of Pfaff's formula to the right-hand side. This is one of the most basic transformation rules for hypergeometric functions and it states

$$
{ }_{2} F_{1}\left(\begin{array}{c}
a, b \\
c
\end{array} \mid x\right)=(1-x)^{-a}{ }_{2} F_{1}\left(\begin{array}{c}
a, c-b \\
c
\end{array} \mid \frac{x}{x-1}\right) .
$$

It can easily be deduced from the integral representation

$$
{ }_{2} F_{1}\left(\begin{array}{cc}
a & b \\
c
\end{array} \mid z\right)=\frac{1}{B(b, c-b)} \int_{0}^{1} t^{b-1}(1-t)^{c-b-1}(1-t z)^{-a} d t,
$$

appearing as entry 9.111 in [6]. In the current problem, this has the positive effect of canceling the fourth powers and converts (7.5) into

$$
{ }_{2} F_{1}\left(\begin{array}{c}
1,2 \\
\frac{1}{2}
\end{array} \mid x\right)=\frac{1}{10}\left[{ }_{2} F_{1}\left(\begin{array}{c}
4,2 \\
\frac{7}{2}
\end{array} \mid \frac{1-\sqrt{x}}{2}\right)+{ }_{2} F_{1}\left(\begin{array}{c}
4,2 \\
\frac{7}{2}
\end{array} \mid \frac{1+\sqrt{x}}{2}\right)\right],
$$

with $z=4 x$.

The proof presented here has been restricted to use only what can be found in the table [6]. Therefore it is natural to search there for hypergeometric identities that look like (7.8). 
There are not so many identities of this type in [6], but fortunately entries 9.136.1 and 9.136.2 give

$$
\begin{aligned}
& { }_{2} F_{1}\left(\begin{array}{c}
2 a, 2 b \\
a+b+\frac{1}{2}
\end{array} \mid \frac{1 \pm \sqrt{x}}{2}\right)=\frac{\Gamma\left(a+b+\frac{1}{2}\right) \sqrt{\pi}}{\Gamma\left(a+\frac{1}{2}\right) \Gamma\left(b+\frac{1}{2}\right)} 2 F_{1}\left(\begin{array}{c}
a, b \\
\frac{1}{2}
\end{array} \mid x\right) \\
& \mp \frac{2 \sqrt{\pi x} \Gamma\left(a+b+\frac{1}{2}\right)}{\Gamma(a) \Gamma(b)}{ }_{2} F_{1}\left(\begin{array}{c}
a+\frac{1}{2}, b+\frac{1}{2} \mid x \\
\frac{3}{2}
\end{array} \mid\right. \text {. }
\end{aligned}
$$

And this is precisely what is needed. To prove (7.8) simply add both cases of (7.9) with the special values $a=2$ and $b=1$. The proof of (7.9) is a direct consequence of a basic hypergeometric identity [3, formula 3.1 .12 , page 128$]$ :

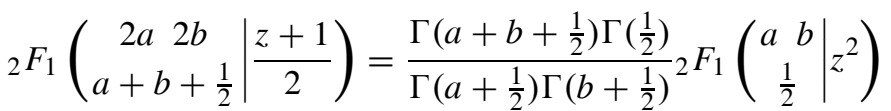

$$
\begin{aligned}
& -z \frac{\Gamma\left(a+b+\frac{1}{2}\right) \Gamma\left(-\frac{1}{2}\right)}{\Gamma(a) \Gamma(b)}{ }_{2} F_{1}\left(\begin{array}{c}
a+\frac{1}{2} b+\frac{1}{2} \\
\frac{3}{2}
\end{array} \mid z^{2}\right) \text {. }
\end{aligned}
$$

The proof of the formula for the generating function of $\left\{1 / C_{n}\right\}$ is now complete.

This section has shown that the evaluation of $f(z)$, the generating function of the reciprocal of Catalan numbers, is equivalent to the identity (7.8). Having established this hypergeometric identity, the evaluation of $f(z)$ has been established. Now it is natural to ask whether it is possible to prove (7.8) by expanding both sides as power series and comparing coefficients of equal powers. A simple calculation, left to the reader, shows that this is equivalent to

$$
\frac{1}{C_{n}}=\sum_{k=2 n}^{\infty} \frac{(4)_{k}(2)_{k}}{5\left(\frac{7}{2}\right)_{k} k ! 2^{k-2 n}}\left(\begin{array}{c}
k \\
2 n
\end{array}\right)
$$

for every $n \in \mathbb{N}$. This brings us back to the reciprocal of Catalan numbers. This is an unexpected turn of events. To finish our discussion, a direct proof of (7.11) is presented next. The authors have been unable to find identities of this type in the literature.

Denote the summand in (7.11) by $f(n, k)$. In order to normalize the sum to start at 0 , write $g(n, k)=f(n, k+2 n)$. Then

$$
\frac{g(n, k+1)}{g(n, k)}=\frac{(k+2 n+2)(k+2 n+4)}{(k+1)(2 k+4 n+7)} .
$$

The representation (3.6) now gives

$$
\sum_{k=2 n}^{\infty} \frac{(4)_{k}(2)_{k}}{5\left(\frac{7}{2}\right)_{k} k ! 2^{k-2 n}}\left(\begin{array}{c}
k \\
2 n
\end{array}\right)=g(n, 0)_{2} F_{1}\left(\begin{array}{c}
2 n+22 n+4 \\
2 n+\frac{7}{2}
\end{array} \mid \frac{1}{2}\right) .
$$

In order to complete the proof, one needs a formula for the hypergeometric function at argument $\frac{1}{2}$. Now Gauss comes to the rescue. The required evaluation is his second sum- 
mation formula (see [3, p. 148], [12, formula 15.4.28], [16, Chapter XIV, Exercise 13])

$$
{ }_{2} F_{1}\left(\begin{array}{c}
a b \\
\frac{1}{2}(a+b+1)
\end{array} \mid \frac{1}{2}\right)=\frac{\Gamma\left(\frac{1}{2}\right) \Gamma\left(\frac{1}{2}(a+b+1)\right)}{\Gamma\left(\frac{a+1}{2}\right) \Gamma\left(\frac{b+1}{2}\right)} .
$$

Replacing in (7.13) and some slight simplification using Euler's duplication formula

$$
\Gamma\left(n+\frac{1}{2}\right)=\frac{(2 n) ! \sqrt{\pi}}{2^{2 n} n !}
$$

yields the desired result (7.11). It is too bad that (7.14) does not appear in [6]. It should definitely be included in the next edition.

\section{Conclusions}

A variety of methods have been used to prove formulas for the generating function of the reciprocal of the Catalan numbers. These methods include traditional proofs, some modern proofs based on algorithms included in symbolic languages and also a proof based only on entries of a classical table of integrals. The authors hoped to illustrate the usual ways in which they approach a problem.

Acknowledgment. The authors wish to thank Armin Straub for providing the proof presented at the end of the last section and also Atul Dixit for many discussions on this problem. The fourth author acknowledges the partial support of NSF-DMS 1112656. The second and third authors are graduate students, partially supported by the same grant. The work of the last author was partially funded by the iCODE Institute, a research project of the Idex Paris-Saclay.

\section{References}

[1] M. Albano, E. Beyerstedt, and V. Moll. The integrals in Gradshteyn and Ryzhik. Part 19: The error function. Scientia, 21:25-42, 2011.

[2] G.E. Andrews. The death of proof? Semi-rigorous Mathematics? you've got to be kidding! The Mathematical Intelligencer, 16:16-18, 1994.

[3] G.E. Andrews, R. Askey, and R. Roy. Special Functions, volume 71 of Encyclopedia of Mathematics and its Applications. Cambridge University Press, New York, 1999.

[4] G. Boros and V. Moll. Irresistible Integrals. Cambridge University Press, New York, 1st edition, 2004.

[5] T. Cadwallader-Olsker. What do we mean by Mathematical Proof? Journal of Humanistic Mathematics, $1: 33-60,2011$.

[6] I.S. Gradshteyn and I.M. Ryzhik. Table of Integrals, Series, and Products. Edited by D. Zwillinger and V. Moll. Academic Press, New York, 8th edition, 2015.

[7] A. Jaffe and F. Quinn. 'Theoretical Mathematics': Towards a cultural synthesis of Mathematics and Theoretical Physics. Bull. Amer. Math. Soc., 29:1-13, 1993.

[8] A. Jaffe and F. Quinn. Response to comments on 'Theoretical Mathematics'. Bull. Amer. Math. Soc., 30:208-211, 1994.

[9] T. Koshy. Catalan Numbers with Applications. Oxford University Press, 2009. 
[10] D.H. Lehmer. Interesting series involving the central binomial coefficient. Amer. Math. Monthly, 92:449457,1985

[11] H.P. McKean. Probability. The Classical Limit Theorems. Cambridge University Press, New York, 2014.

[12] F.W.J. Olver, D.W. Lozier, R.F. Boisvert, and C.W. Clark, editors. NIST Handbook of Mathematical Functions. Cambridge University Press, 2010.

[13] B. Sury, T. Wang, and F. Zhao. Identities involving reciprocals of binomial coefficients. Journal of Integer Sequences, 7:Article 04.2.8, 2004.

[14] W.P. Thurston. On proof and progress in Mathematics. Bull. AMS., 30:161-177, 1994.

[15] C. Vignat and V. Moll. A probabilistic approach to some binomial identities. Elem. Math., 69:1-12, 2014.

[16] E.T. Whittaker and G.N. Watson. Modern Analysis. Cambridge University Press, 1962.

[17] D. Zeilberger. Theorems for a price: Tomorow's semi-rigorous mathematical culture. Notices AMS, 40:978-981, 1993.

T. Amdeberhan, X. Guan, L. Jiu and V.H. Moll

Department of Mathematics

Tulane University

New Orleans, LA 70118, USA

e-mails: tamdeberhanatulane. edu

$$
\begin{aligned}
& \text { xguan1@tulane. edu } \\
& \text { 1jiu@tulane.edu } \\
& \text { vhm@tulane. edu }
\end{aligned}
$$

\section{Vignat}

Department of Mathematics

Tulane University

New Orleans, LA 70118, USA

and

Supelec L.S.S.

Université Paris Sud Orsay, France

e-mail: cvignatatulane.edu 